\title{
Smart Grid and Customer Transactions: The Unrealized Benefits of Conformance
}

\author{
Girish Ghatikar \\ Deputy Leader, Grid Integration \\ Lawrence Berkeley National Laboratory \\ Berkeley, CA 94720. U.S.A \\ Ed Koch \\ Senior Fellow \\ Honeywell Building Solutions \\ Golden Valley, MN 55422. U.S.A
}

\author{
Jim Zuber \\ Chief Technology Officer \\ QualityLogic, Inc. \\ Simi Valley, CA 93065. U.S.A \\ Rolf Bienert \\ Technical Director \\ OpenADR Alliance \\ Morgan Hill, CA 95037. U.S.A
}

\begin{abstract}
Smart Grid investments and research promise significant benefits to both customers and the grid. One major benefit is data and communications that enable customer-side resources to participate in electricity programs-i.e., transact with the electric grid. The U.S. Smart Grid activities and investments, which account for nearly three billion dollars in publicly documented expenses, build on interoperability standards activity. Standards interoperability for grid transactions can be viewed from two key interfaces - between (1) Smart Grid domain interfaces, and (2) customer-side resources and the electric grid. Conformance testing and certification is key to ensuring that standards are interoperable and secure. This paper describes the conformance development and certification process for a key communications standard-Open Automated Demand Response (OpenADR) - as an interface between customers' systems and electricity service providers, to enable demand response and price transactions. Through this process, we describe the benefits of conformance to support low-cost automated DR (AutoDR) and customer-side transactions within the distribution electric grid. The paper evaluates examples of applications and further developments for emerging transactive energy concepts that can be used to scale existing systems and to leverage the investments and support compliance to any building codes and appliance standards. The conformance process can be the key to realizing interoperability benefits and market acceptance. Conformance is also a key to enabling native DR and price transaction capabilities in customers' systems; a feature that can be replicated for diffusion and can lower DR technology costs by a significant margin.
\end{abstract}

Keywords - Smart Grid Transactions; Innovation Cycle; Open Standards; Interoperability; Conformance

\section{INTRODUCTION AND BACKGROUND}

Smart Grid investments and demonstrations promise significant economic benefits to both customers and the grid. One such activity looks at data and communications to help enable customers and customer-side resources to participate in electricity programs-i.e., transact with the electric grid. The U.S. Smart Grid activities and investments in its deployment account for nearly three billion dollars in publicly documented expenses [1]. These investments leverage the interoperability standards activities coordinated by the National Institute of Standards and Technology (NIST). Customers' Smart Grid interoperability can be viewed for two key interfaces - between (1) Smart Grid domain interfaces, and (2) customer-side resources and the electric grid. Figure 1 shows these two interfaces where system interoperability is necessary. The standards conformance testing and certification can be leveraged as a key process to ascertain that systems are interoperable and secure between these key domains. While conformance tests ascertain if the product conforms to a standard, conformance testing in itself does not assure that an implementation based on the standard is interoperable and secure. Additional tests can be included in the conformance testing to ensure systems interoperability and cyber-security.

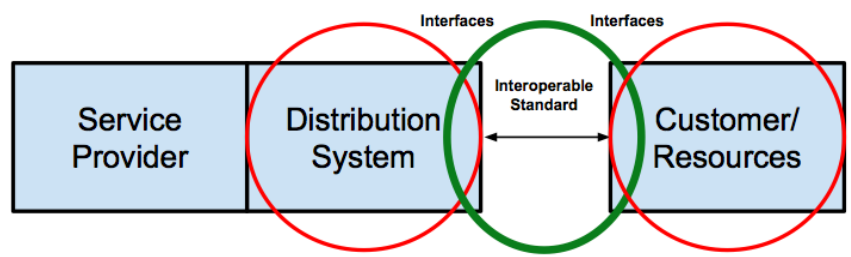

Fig. 1. Standard Interfaces for Smart Grid Domains - Distribution Systems and Customer/Resources

This paper describes the conformance and certification development for an open Smart Grid interoperability standard -Open Automated Demand Response (OpenADR) - as an interface between customers and the electricity service providers for demand response and price transactions. The focus is on conformance development for interoperability between customer-side systems (e.g., energy management control systems) and loads (e.g., heating/cooling, lighting, appliances) and the electric grid. The paper describes the benefits of conformance to support interoperability and lowcost automated DR (AutoDR), as well as new emerging concepts for customer-side transactions with the electric grid.

\section{A. Interoperable Standards for Automated Demand Response}

The NIST-coordinated Smart Grid Interoperability Panel (SGIP) provides a Catalogue of Standards for standard demand response (DR) and distributed energy resource (DER) signals that are vetted through the national Smart Grid standards framework [2]. A key goal of this NIST activity is to enable interoperability of customer-side resources and systems with the electric grid signals. This enables customers to participate in DR programs, dynamic pricing, and future transactive energy markets to modify the electric load profiles during times of supply and price changes [3]. One such communications standard, Open Automated Demand Response (OpenADR), is based on a client/server model, where the DR service provider conveys signals to customers. OpenADR has evolved from a communication specification, OpenADR 1.0, which is implemented in utility AutoDR programs, to a formal standard, OpenADR 2.0. OpenADR 2.0 clients (subscribing to signals) are generically called virtual end nodes (VEN), and the servers (publishing the signals) are generically called virtual top nodes (VTN) [4]. OpenADR 2.0 VTN/VEN pairs 
provide a platform for electric grid operators, customers, and markets to enable interoperable DR and DER signals. In California, OpenADR 1.0 is commercially deployed in more than 1,300 facilities, for a total DR load of about 250 megawatts (MW), with technology enablement costs ranging from $\$ 170 /$ kilowatt $(\mathrm{kW})$ to $\$ 300 / \mathrm{kW}$ [5]. The conformance described here addresses the set of technical and OpenADR 2.0 profile specification requirements for interoperability and security testing. We look at the OpenADR conformance process, with emphasis on the following:

1. Conformance and test tool development

2. Interoperability and cyber security testing and certification

The lessons from the conformance development can be used by other standards, transferred to organizations and regulators for adoption in policy and building codes, and foster the customer-side automation for participation in Smart Grid transactions. The outcomes from this study will provide a framework for other standards bodies and public-private stakeholders involved in Smart Grid activities. Development of conformance rules are essential for certification of such standards-based messaging protocols, and they play a key role in: (a) the California Energy Commission's (CEC's) Title 24 building code for AutoDR [6], (b) the California Public Utilities Commission's (CPUC) Rule 21 to enable customerside DER to participate in the electric grid program [7], and (c) CPUC Rule 24 (under finalization), which enables direct participation of retail customers and their resources in the wholesale DR markets [8]. Conformance enables the existence of multiple access levels for customers of wholesale and retail DR markets by providing common standardized interfaces.

\section{B. Background}

The conformance testing and certification process for OpenADR 2.0 was developed in close coordination with relevant industry experts, starting with standards organizations, utilities, the Independent System Operator / Regional Transmission Organization (ISO/RTO) Council (IRC), and other national Smart Grid committees (e.g., Smart Grid Testing and Certification Committee) [9]. This development and adoption cycle is based on over ten years of research and deployment experience of low-cost automation and open communication technologies for AutoDR. This is best described using the innovation cycle in Figure 2, which is adapted from the innovation system in an earlier study [10]. The development and adoption of interoperable AutoDR technologies can be viewed as four interrelated components of invention (idea, knowledgebase, prototypes), translation (commercial product or process), adoption (initial deployment of new technology), and diffusion (increased adoption and use). Before the final scaled diffusion, feedback streams enable incremental technology improvement. At present, OpenADR 2.0 is at an adoption stage. The conformance framework and deployment of OpenADR 1.0 sets OpenADR 2.0 in a low-risk, high-benefit pathway toward diffusion of Smart Grid systems.

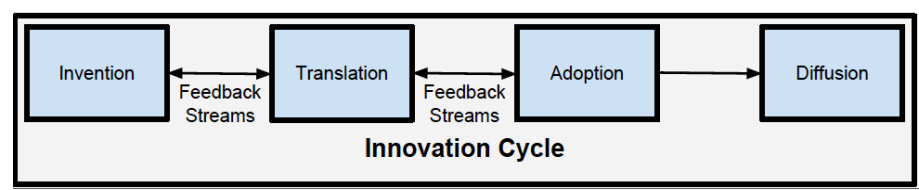

Fig. 2. Innovation Cycle: Invention, Translation, Adoption, Diffusion

As the technology is ready for diffusion to meet the current market needs, the new research areas, such as energy transactions across Smart Grid domains, push the boundaries to further develop the existing technology to support new market needs for economic transactions [11].

Transactive Energy (TE) is not a standard, but a technique whereby the consumption or flow of electric power (buying/selling) is managed using economic or market-based constructs. Energy transactions and/or operational decisions are based upon a value that represents the relative or actual economic value associated with the energy and its delivery. Transactive Control (TC) is a specific case of the general class of techniques referred to as TE. The focus of TC, as the name implies, is on making operational "control" decisions based upon a "value," which may represent purely economic value or some more abstract notion of value that could encompass factoring in operational concerns. The Pacific Northwest Smart Grid Demonstration Project (PNWSGDP) is demonstrating the TE and TC concepts [12]. One study links OpenADR standards with TE and TC, and explores the relationships with its source, Energy Interoperation 1.0 (EI 1.0) [13]. One interesting conclusion was that OpenADR could play a potentially complementary role in the adoption of TC.

In the broadest context, TE could be considered by any current technology where the decision to participate in an energy transaction or make operational decisions is based upon a value representative of economic or operational value. OpenADR applications to TE might include participation in the wholesale electricity markets based upon DR load change requests or electricity pricing information.

\section{Paper Organization}

The paper is organized as follows: Section II describes conformance rules and test tool development that includes selection of transport mechanisms and cyber security schemes, and profiling the services for OpenADR 2.0. Section III describes the testing and certification procedures to ascertain the interoperability and cyber-security of OpenADR 2.0 VTN and VEN, including the adherence to national interoperability guidelines. Section IV lists discussion topics, conclusions, and future work for OpenADR 2.0 and its links to TE concepts.

\section{CONFORMANCE AND TEST TOOL DEVELOPMENT}

This section describes the process involved for the following activities: (1) selection of transport mechanisms and cyber security; (2) specification profiling and validation; and (3) selection of serviced and cyber security. 


\section{A. Conformance Scope}

To give a perspective and understanding of the conformance scope for OpenADR 2.0, it necessary to understand how different standards fit within the Open System Interconnection Reference Model (OSI Model). ${ }^{1}$ OpenADR is primarily a Layer 7 or "Application Layer" specification in reference to the seven-layer OSI Model. The other areas between Layer 5 to 7, such as security requirements and sessions, may apply to OpenADR. However the scope of conformance framework for standards is limited to the Application Layer. The selection of suitable transport and security layers (Layers 4 to 7 ) is necessary to test OpenADR-compliant clients and certify their communication abilities for on-field deployments. Since the servers that publish DR or price signals and the clients that subscribe to these signals can evolve independently, one has to determine the type of transport schemes that are required for interoperability testing. The OpenADR Alliance, a nonprofit industry consortium, manages the development of conformance rules and certification for OpenADR 2.0. The OpenADR Alliance comprises key DR market stakeholders such as research organizations, utilities, systems operators, and vendors that develop interoperable products. The OpenADR Alliance is not a standards development organization (SDO), but instead relies on standards created by other SDOs.

\section{B. Selection of Transport Mechanisms and Cyber Security}

Table 1 summarizes the different SDO specifications for transport data models, transport mechanisms, and cyber security, and their uses in OpenADR 2.0:

TABLE 1. DATA MOdELS, Transport MECHANISMS, AND SECURITY APPLICATIONS FOR OPENADR 2.0

\begin{tabular}{|c|c|c|}
\hline SDO & Specification & Use \\
\hline $\begin{array}{l}\text { Organization for } \\
\text { Advancement of } \\
\text { Structured Information } \\
\text { Standards (OASIS) } \\
\text { Energy Interoperation } \\
\text { (EI) Committee }\end{array}$ & $\begin{array}{l}\text { EI } 1.0 \text { based on } \\
\text { eXtensible Markup } \\
\text { Language (XML) }\end{array}$ & $\begin{array}{l}\text { Data models for } \\
\text { exchanging } \\
\text { application-level } \\
\text { messages }\end{array}$ \\
\hline $\begin{array}{l}\text { Internet Engineering } \\
\text { Task Force (IETF) }\end{array}$ & $\begin{array}{l}\text { Hypertext Transfer } \\
\text { Protocol (HTTP) }\end{array}$ & $\begin{array}{l}\text { Application-level } \\
\text { transport }\end{array}$ \\
\hline $\begin{array}{l}\text { XMPP Standards } \\
\text { Foundation (XSF) and } \\
\text { Internet Engineering } \\
\text { Task Force (IETF) }\end{array}$ & $\begin{array}{l}\text { XML Messaging } \\
\text { and Presence } \\
\text { Protocol (XMPP) }\end{array}$ & $\begin{array}{l}\text { Application-level } \\
\text { transport }\end{array}$ \\
\hline $\begin{array}{l}\text { Internet Engineering } \\
\text { Task Force (IETF) }\end{array}$ & $\begin{array}{l}\text { Transport Layer } \\
\text { Security (TLS) } 1.2\end{array}$ & $\begin{array}{l}\text { Communication } \\
\text { security protocol }\end{array}$ \\
\hline $\begin{array}{l}\text { Internet Engineering } \\
\text { Task Force (IETF) }\end{array}$ & $\begin{array}{l}\text { Public Key } \\
\text { Infrastructure (PKI) } \\
\text { and Certification }\end{array}$ & $\begin{array}{l}\text { OpenADR PKI } \\
\text { policy } \\
\text { framework }\end{array}$ \\
\hline $\begin{array}{l}\text { Internet Engineering } \\
\text { Task Force (IETF) }\end{array}$ & $\begin{array}{l}\text { Hypertext Transfer } \\
\text { Protocol (HTTP) }\end{array}$ & $\begin{array}{l}\text { Application-level } \\
\text { transport }\end{array}$ \\
\hline
\end{tabular}

In Table 1, it should be noted that OpenADR 2.0 leverages and relies on a suite of existing and open standards that are typical

\footnotetext{
${ }^{1}$ The OSI model is a telecommunications systems framework based on the 7-layer hierarchical stack referenced across the industry. It allows the interoperability between communications protocols.
}

of any application that communicates via the Internet. This means that there are existing tool sets and implementations that have already been validated to be conformant with those standards, and the OpenADR Alliance therefore focuses on defining and testing conformance against the application-level messages that rely on OASIS EI 1.0 standards.

In addition, OpenADR 2.0 allows vendors to pick and choose among various options to support different deployments. For example, vendors can choose among a variety of security standards to meet utility and customer requirements, and in addition, they can choose between HTTP or XMPP as the transport protocol, depending upon how their products are developed and what the requirements are for how the customers interact with the utility or the system operator. For example, XMPP is generally more appropriate for lowerlatency communications. In general, such variability and optional elements in implementations is problematic for interoperability, but the way that OpenADR 2.0 deals with this is by requiring that all servers (VTNs) support a superset of features, while individual clients (VENs) have the flexibility of picking and choosing which features they will support. Optionality in both VTN and VEN is problematic because there might be no matching supported services at the either end, leading to non-conformity. Since OpenADR interoperability is in the communications between VTNs and VENs, and not in the communications between VENs, this ensures that any VEN can communicate with any VTN. This is similar to how other industries, such as cellular communications, deal with variability among end-use devices.

\section{Specification Profiling and Validation}

One of the main conformance activities was to profile the OASIS EI 1.0 specification and define conformance rules and test procedures against that profile. This was necessary, as the goals of OASIS EI 1.0 standards go beyond DR and price communications. The profiling of the OASIS EI 1.0 specification had the following objectives:

- Reduce the complexity and payload sizes of EI 1.0 by using only those elements required for DR and prices.

- Where possible, reduce variability by constraining the values of elements in the EI 1.0 data models.

- Extend the EI 1.0 data models when necessary.

- Create profiles to support different implementation and deployment scenarios.

To create implementation profiles, we decided to create two separate key profiles: A and B, wherein A is a subset of the B profile. Profile A was intended to offer a minimum set of functionality to support simple communications, smaller payloads, and resource-constrained devices. A fully featured profile B supports complex communications, reporting services, and resource-intensive devices. Figure 3 shows the relationship between the profiles and the OASIS EI 1.0. In addition, a reporting-only profile allows VENs with reportingonly capabilities to provide services to the VTN. Such a reporting-only VEN can be used in combination with an A profile to support reporting capabilities. 


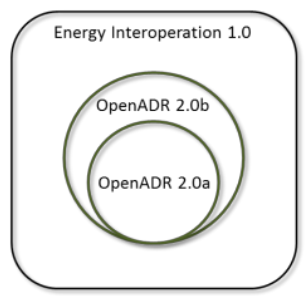

Fig. 3. OASIS EI 1.0 Standard and OpenADR 2.0 Profile Relationships

The formal implementation requirements for each OpenADR profile are documented in an XML schema and in a set of conformance rules in the profile specification document [4]. The conformance rules specify protocol behavior requirements that cannot be captured by the schema, as well as requirements specific to the transport and security protocols. The A profile contains 59 conformance rules, and the B profile has 147 rules.

The Protocol Implementation Conformance Statement (PICS) extracts the testable requirements from the schema and profile conformance rules, and is used by vendors as a checklist to ensure all the necessary requirements are implemented to ensure interoperability. A PICS document is submitted as part of the certification process as an assertion that all the requirements have been meet. The PICS is also the basis for a set of certification test cases covering each of the testable requirements for a given profile. The test cases are run using a test harness that simulates the behavior of the counterpart in a message exchange. Running a test case results in a "pass" or "fail" result and validates a specific set of requirements. An implementation that passes all the test cases can be formally certified. An example of the relationship between the conformance rules, PICS, and test cases would be as follows:

- Conformance rule 47 states: "An event with an overall duration of 0 indicates an event with no defined end time and will remain active until explicitly cancelled."

- The PICS asks the implementer to confirm that they have implemented conformance rule 47.

- Test case A_Ex_0250 validates that conformance rule 47 has been implemented correctly.

The OpenADR payloads used for exchanging events, reports, schedules, and other operational characteristics are defined in the OpenADR schema. All of the core schema objects used by OpenADR were derived from the Energy Interoperation schema, and those objects maintain conformance with Energy Interoperation schema.

\section{Selection and Support of, Services, and Cyber Security}

Table 2 summarizes the supported services and functions, transport protocols, and security levels for conformance certification of the VTN, as well as A and/or B profiles of the VEN. These are described in detail in the OpenADR 2.0 profile specifications document [4]. The optional elements are only allowed for the VENs, thus enabling their interoperability with any VTN. The following services from OASIS EI 1.0 are supported in OpenADR 2.0 Profile Specifications-EIEvent, EIOpt, EIReport, and EIRegisterParty. The descriptions of these services are well described in the OASIS EI 1.0 standard
[13]. As an example, the simple profile A supports a subset of EIEvent services.

With respect to transport protocols, the VEN has a choice of using either simple HTTP or XMPP. The basic XML payloads are the same for either protocol. In general, HTTP is more broadly adopted within tool chains in use today, but XMPP is increasing in adoption and may be a more appropriate protocol for low-latency delivery of event information, since it can be easier to get through firewalls using XMPP when the VTN is initiating communications with the VEN.

TABLE $2 . \quad$ OPENADR 2.0-SUPPORTED SERVICES, TRANSPORT PROTOCOLS, AND SECURITY CERTIFICATION LEVELS

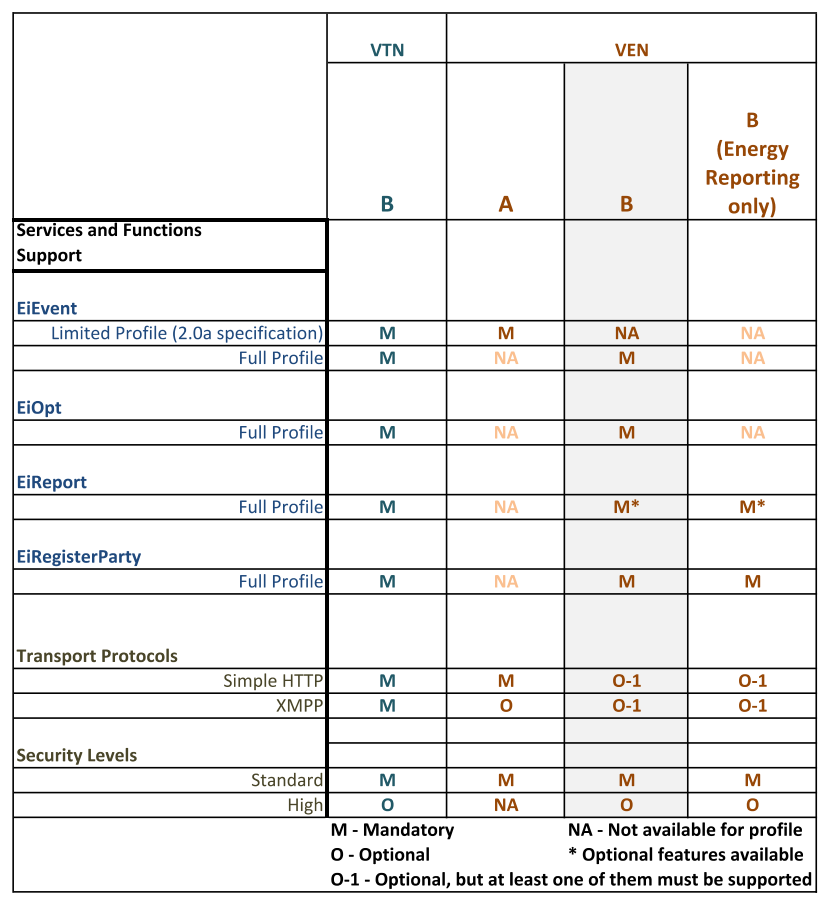

In addition to the protocols above, the VEN may use a PUSH or PULL model to interact with the VTN. In the PULL model, the VEN initiates all communications with the VTN (i.e., pulls the information); while in the PUSH model, the VTN may initiate communications with the VEN. The PULL model is typically easier to configure at the VEN site, since it can be done without reconfiguring the customer's firewall, which may be required to support the PUSH model. While the standard security is mandatory, the higher level of security with XML data encryption is optional for both VTN and VEN, as any security level is dependent on the DR program requirements.

For security, the OpenADR Alliance consulted with security experts and considered the cyber security guidelines defined by the National Institute of Standards and Technology Report (NISTR) 7628 [14]. The NISTR 7628 is an important document that provides cyber security guidelines for the energy sector and Smart Grid interoperability standards. NISTR 7628 also maps the North American Electricity Reliability Corporation's (NERC) Critical Infrastructure 
Protection (CIP) requirements. Furthermore, the overall process from profiling the existing standards to creating the certification program followed the SGIP's Interoperability Process Reference Manual (IPRM) [15]. The IPRM describes industry practices that lead to the creation of interoperable system specifications by creating adequate conformance statements and test processes. This selection of security and transport mechanism, and the subsequent conformance development process through PICS and test tools, provides an infrastructure to test and certify both clients and servers for conformance and interoperability.

\section{INTEROPERABILITY AND CYBER SECURITY TESTING AND CERTIFICATION}

This section describes the testing and certification of OpenADR 2.0 servers and clients, to ensure that they interoperate and that the infrastructure supports present U.S. programs for DR and price transactions - and potentially, for future electricity markets. OpenADR supports generic program requirements and different signaling infrastructure support to accommodate multiple timescale needs for DR and price transactions of customer's resources. The service providers usually define these program requirements to facilitate the participation of customer resources. These timescale needs are defined between the notification time of the signal, when the resources must be ready to provide grid services (start time) and for what duration (end time), and the time these resources return to their normal state (recovery). For example, the notification time for an ancillary services program may be 30 minutes or less with event duration of 10 minutes. The recovery period will indicate the availability of the resource(s) for the next market cycle. The signaling infrastructure includes simple signals such as pending, active, and completed states for different levels, and complex information such as load change requests or price information for different intervals (e.g., day-ahead hourly prices and day-of hourly prices).

\section{A. Certification Procedure}

All service providers and technology vendors who wish to have their products identified as OpenADR compliant have to go through a testing and certification process. Once certified, the vendors add the "OpenADR certified" logo to their product and marketing materials so that the customers know the system or device is certified to be OpenADR compliant. This is critical from the perspective of interoperability, which guarantees that any VEN (client) can interoperate with the VTN (server). In addition, it enables decoupled development of AutoDR-enabled clients from the DR program design. Certification is an endorsement of compliance. Without it there is no assurance that the VEN and VTN interoperate.

For certification, OpenADR 2.0 VENs and VTNs must pass a formal certification test at an ISO 17025 accredited test facility. This accreditation is important for quality assurance and to meet the SGIP IPRM requirements, thus enabling OpenADR 2.0 to meet one of the eligibility requirements for interoperability. The test tools, developed by QualityLogic ${ }^{\circledR}$, are based on a test specification developed by the OpenADR
Alliance that documents approximately 200 test cases for profile A and 400 test cases for profile B. To facilitate this, a certification test harness or tool was developed that implements each of the defined test cases. This test harness is used during the certification process to validate that profile $\mathrm{A}$ and/or profile B devices conform to the OpenADR profile specifications.

Each test case exercises a specific service interaction pattern based on typical use cases. In the aggregate the test cases validate each of the testable requirements defined in the PICS. The following conformance characteristics are validated each time a test case is run: (a) payloads contain well-formed XML; (b) payloads validate against the OpenADR schema; (c) a correct message interaction pattern occurs; (d) OpenADR conformance rules are followed; and (e) the intent of the test case is achieved.

\section{B. National Interoperability Guidelines}

The development of the test specification and the test harness for certification followed the guidelines outlined in the aforementioned IPRM and the NISTR 7628 cyber security guidelines. Such requirements are needed to ensure that the implementation of a specific standard by users meets the base case requirements defined for national or international implementations.

The OpenADR Alliance's testing requirements for interoperability is recognized as the most complete implementation of this reference manual. The test harness is made available to OpenADR 2.0 implementers for precertification testing, which enables vendors to have confidence they will pass the certification process offered by an accredited test lab once they complete their internal development and testing. The testing is conducted for OpenADR VTN or VEN software. Such testing for compliance against the base standard is called the "implementation under test (IUT)," which once certified, could be replicated in many vendors' hardware-based devices and/or systems.

The IUT must pass certification to obtain production x.509 public key infrastructure (PKI) security certificates. This procedure is an effective cyber security enforcement mechanism during the certification process. Once certified, manufacturers can establish an account with the recognized certificate authority (CA), which is managed by NetworkFX ${ }^{\circledR}$. The VTN and VEN certificates using different public cryptography mechanisms, such as Rivest, Shamir, and Adleman (RSA) or elliptic curve cryptography (ECC) mechanisms, can be obtained to implement them in final installations. These public cryptography mechanisms are required by the aforementioned NISTR 7628 cyber security guidelines. The full cost of certification varies greatly by the number of purchased units or how the IUT is replicated in scale. It ranges from $\$ 1.60$ per client certificate for very low volumes (dozens to hundreds) to $\$ 0.18$ per client for high volumes (hundreds to thousands). Server certificates cost approximately $\$ 275$. While the OpenADR Alliance encourages 
the use of their specific digital certificates for interoperability and certificate management reasons, individual DR programs might also opt to use different kinds of certificates from other certificate authorities.

\section{Device and System Certification}

Any hardware or software implementation that exchanges OpenADR payloads with an OpenADR 2.0 VEN or VTN should be certified. The OpenADR implementation may take the form of a stand-alone software in a gateway device, a software function within an energy or building management system, as an embedded module in load-consuming devices such as lighting fixtures or appliances, or as part of large, complex back-end software systems such as a enterprise DR management systems. All of these OpenADR 2.0 implementations require certification, and the test house providing these certification services has experience with most of these types of OpenADR implementations. To ensure consistent test results, the OpenADR Alliance started the certification program with one test house, Intertek ${ }^{\circledR}$. In 2014, another test house, TÜV Rheinland ${ }^{\circledR}$, was established to cater to the Japanese market. The Alliance will consider additional test service providers as the demand for certification increases.

Currently the OpenADR Alliance's testing and certification program does not take into account specific regulatory requirements for AutoDR in building codes or appliance standards. However, the California Title 24 building code requires HVAC and lighting controls in non-residential facilities greater than 10,000 square feet to install a standardsbased messaging protocol for AutoDR communication interfaces $[6,16]$. Since all California investor-owned utilities (IOUs) require certified OpenADR systems for their OpenADR-based AutoDR programs, the use of certified OpenADR products is implied. There is also an effort by these IOUs to expand these requirements, albeit presently not required by the Title 24, to residential customers (e.g., communicating thermostats). As the implementation of OpenADR-certified devices in AutoDR programs expands, the OpenADR Alliance is establishing testing and program guidelines for specific DR program implementations.

\section{DISCUSSIONS, CONCLUSIONS, AND FUTURE WORK}

In this paper we discussed the benefits of the conformance development process and the testing and procedures for interoperability and cyber security of a national and international Smart Grid standard, OpenADR, for DR and price transactions. In reference to the innovation cycle in Figure 2, it is clear that for adoption and diffusion of interoperable Smart Grid technologies, the conformance process plays a key role. Conformance thus supports critical needs of both grid and customers for interoperability, cyber security, and cost effectiveness. The initial deployments of new market-ready technologies can be scaled for deployments and adoption in commercial programs. The interoperability of interfaces between the distribution systems and customer-side devices and/or systems is critical for the diffusion of Smart Grid technologies. The innovation cycle of invention, translation, and adoption can be repeated to enable commercially deployed technologies to be enhanced for new market design concepts such as transactive energy and transactive controls. We adapted the innovation cycle for OpenADR conformance applications, current developments, and future market trends.

Below, we discuss and offer key conclusions of OpenADR conformance development and discuss future research to take place while the existing Smart Grid investments take shape.

\section{A. Discussions}

The success of a new Smart Grid technology can be evaluated by its potential for diffusion, ease of integration with Smart Grid domain interfaces, and ease of support for new electricity market concepts. Here we discuss OpenADR in the context of commercial deployments, interoperability between distribution systems and customer domain interfaces, and scaling the infrastructure to new emerging concepts such as TE and TC.

Commercial Program Deployments: As of 2013, the California IOUs' AutoDR programs began to require OpenADR 2.0 certification for both commercial and industrial customers, including aggregators. Such requirements are being considered for residential programs as well. In a typical process, once a customer is enrolled in an AutoDR program, the certified VEN(s) would be installed and registered to a certified VTN. After the communication interoperability is tested between the customer VEN(s) and the utility VTN, the customer enrolls for participation. The following steps are used for customer enrollment and VEN registration in the IOUs' AutoDR programs. Here, step 2 implies that the customer's VEN is tested and certified for interoperability. This process can be an important element for functional testing of AutoDR compliance to building codes such as California Title 24 .

1. Device Installation: Select and install a certified VEN to enable participation in a utility AutoDR program.

2. Communication Tests: Test that the VEN can receive the OpenADR 2.0-compliant signal from the VTN.

3. Controls Integration: Program controls for the integration of AutoDR strategies to automatically receive and respond to standardized OpenADR signals.

4. End-to-End Automation Validation: Evaluate controls systems' automated response to OpenADR signals (e.g., $\mathrm{kW}$, price), and measure and verify load changes.

Interoperability Between Distribution System and Customer Interfaces: Figure 1 shows the need for interoperable interfaces between Distribution Systems and Customer/Resources. Here we provide examples of such developments for OpenADR. For interfaces with distribution systems, the International Electrotechnical Commission (IEC) Project Committee 118 is working on the interoperability between the IEC Common Information Model (CIM) with OpenADR 2.0 VTN using an OpenADR 2.0 publicly available specification [17]. The interfaces for Customer/Resources are based on the earlier findings from OpenADR adoption, where the existing facility protocols and systems interoperate with an 
OpenADR VEN through a gateway (hardware) interface, or natively within a controls system as a logical (software) interface [18]. For OpenADR 2.0 diffusion, one must consider full interoperability with other facility-centric standards, such as the facility Smart Grid information model and other control system protocols [19].

Support for Transactive Energy and Transactive Controls: While the OSI model describes the scope of conformance for OpenADR and its relationship to different network layers, the GridWise $^{\mathrm{TM}}$ Architecture Council's (GWAC) interoperability context-setting framework (or "GWAC stack") is an important link to the emerging TE and TC concepts [20]. The GWAC, with support from the U.S. Department of Energy (DOE) and the engagement of SGIP members, is coordinating a national effort to define a common framework for TE and the GWAC stack. It is important to link OpenADR with the GWAC stack for future OpenADR applications to emerging TE and TC concepts. Although the GWAC stack describes eight layers of interoperability, the following four layers are relevant to OpenADR: (1) network interoperability, (2) syntactic interoperability, (3) semantic understanding, and (4) business context. Table 3 summarizes the adaptation of the OpenADR relationship from an earlier study [21].

TABLE 3. DATA MODELS, TRANSPORT MECHANISMS, AND SECURITY APPLICATIONS FOR OPENADR 2.0

\begin{tabular}{|l|l|}
\hline \multicolumn{1}{|c|}{ SDO } & \multicolumn{1}{c|}{ Specification } \\
\hline $\begin{array}{l}\text { Business } \\
\text { Context }\end{array}$ & $\begin{array}{l}\text { OpenADR 2.0 inherits the business context } \\
\text { from the EMIX and EI standards. }\end{array}$ \\
\hline $\begin{array}{l}\text { Semantic } \\
\text { Understanding }\end{array}$ & $\begin{array}{l}\text { The publicly available schema and } \\
\text { specification describe the semantic meaning of } \\
\text { data contained in XML data structures. }\end{array}$ \\
\hline $\begin{array}{l}\text { Syntactic } \\
\text { Interoperability }\end{array}$ & $\begin{array}{l}\text { The schema defined in the XML standard } \\
\text { defines the data models and message payloads. }\end{array}$ \\
\hline $\begin{array}{l}\text { Network } \\
\text { Interoperability }\end{array}$ & $\begin{array}{l}\text { Supported by transport mechanisms where key } \\
\text { IP-based transports and interactions between } \\
\text { the participants are defined. }\end{array}$ \\
\hline
\end{tabular}

Since OpenADR as a standard defines the data models to support the application layer, the conformance assures compliance of XML syntax for interactions with key network/transport layers. While OpenADR facilitates DR and price transactions for different market contexts, the utilities or system operators define its link at the higher levels of the GWAC stack, such as business procedures and objectives.

\section{B. Conclusions}

Conformance is important for the adoption and diffusion of Smart Grid technologies by customers, industry, markets, and regulatory mechanisms. Conformance is key to realizing market acceptance and cost effectiveness. Conformance for OpenADR ensures that the customer-side systems, and utility and/or system operators' grid signals can interoperate and are secure. This will enable the decoupling of technology development and deployment of customer-side resources for grid transactions and enable technologies to be accessible for Smart Grid investments. OpenADR conformance enables native transaction capabilities in customers' devices and/or systems, a feature that can be replicated in scale to lower the technology enablement costs and enable compliance to building codes such as California Title 24. The lessons from conformance development and testing and certification procedures can be applied to other standards. Conformance offers some key benefits:

- It enables market acceptance through interoperability of key Smart Grid standards for both DR service providers' distribution systems and customers' systems.

- It manages optionality of Smart Grid standards and enables client and server interoperability and decoupled implementation of customer-side technologies.

- It offers an interoperability platform, low-cost automation, and applications to the policy objectives.

\section{Future Work}

We list two key future recommendations that will not cause any impact to ongoing adoption and diffusion efforts. The first recommendation optimizes the OpenADR payload and the second recommendation offers extensions for emerging $\mathrm{TE}$ and TC concepts. These recommendations must be implemented after detailed performance assessment of ongoing field deployments and making sure that the backward compatibility to OpenADR 2.0 is retained.

Schema Improvements for OASIS EI 1.0: The OASIS EI 1.0 standard must consider payload size reduction to support dataconstrained networks and devices. We suggest the following updates to EiReport service to identify available resources for $\mathrm{DR}$, and to enable transactions by allowing VEN to communicate the availability of load (e.g., $500 \mathrm{~kW}$ is available when $750 \mathrm{~kW}$ is requested at a specific time):

- Enable VTN to request a report associated for a specific event by adding the event identification to the report payload. This will allow identification of customer's resources (and not just VEN) that participate in an event and also the status of these resources.

- To support reporting services for aggregation, enable the aggregation flag in the EI 1.0 standard in the report metadata. This will create a single aggregate report or individual report for each resource.

- Offer selective addition or deletion of reports, without the need to transmit the entire report metadata.

Extending OpenADR for TE and TC: While this is not an immediate conformance need, the following enhancements to the existing EI 1.0 standard would allow customers to support the TE services and concepts, leveraging the existing products:

- To enable a full-scale TE, as demonstrated in the PNWSGDP, extend OpenADR 2.0 to support peer-topeer communications (a VEN talking to another VEN).

- When TE is ready for adoption, make VTN a mandatory report provider, which is a logical way to exchange symmetrical transactive signals beyond customer DR and price transactions. This is currently optional.

- Enable OpenADR 2.0 reports to be sent at both fixed (existing feature) and periodic bursts to enable transactive systems to interact when needed. 


\section{ACKNOWLEDGMENTS}

The work described in this report by the LBNL author was coordinated by the Demand Response Research Center and funded by the California Energy Commission (Energy Commission), Public Interest Energy Research (PIER) Program, under Work for Others Contract No. 500-03-026, and by the U.S. Department of Energy under Contract No. DE-AC02-05CH11231. The authors acknowledge the California Energy Commission and the PIER Program staff for their continued support.

\section{REFERENCES}

[1] U.S. Department of Energy (DOE), Office of Electricity Delivery and Energy Reliability, "Economic impact of Recovery Act investments in the Smart Grid, Smart Grid Investment Grant Program,” April 2013.

[2] National Institute of Standards and Technology (NIST), U.S. Department of Commerce, "NIST framework and roadmap for Smart Grid inteoperability standards, Release 2.0," February 2012.

[3] U.S. Department of Energy (DOE), "Benefits of demand response in electricity markets and recommendations for achieving them," A report to the U.S. Congress, February 2006.

[4] OpenADR Alliance, "OpenADR 2.0 Profile Specifications, A and B Profiles," July 2011 and 2013.

[5] G. Ghatikar, D. Riess, and M. A Piette, "Analysis of Open Automated Demand Response deployments in California and guidelines to transition to industry standards," LBNL-6560E, January 2014.

[6] A. Gonzalez, H. Hauenstein, G. Ghatikar, and P. Eilert, "Unlocking the Smart Grid through building codes and communication standards: Code opportunities to increase DR transactions," 2014 American Council for an Energy-Efficient Economy summer study (in press).

[7] California Public Utilities Commission Rule 21, "Issues, priorities, and recommendations for energy storage interconnection,” July 2014

[8] California Public Utilities Commission (CPUC) Draft Rule 24, "Direct Particpation of Demand Response." (unpublished)
[9] G. Ghatikar, and R. Bienert, "Smart Grid standards and sysetms interoperability: A precedent with OpenADR," Proceedings of Grid Interop, LBNL-5273E, December 2011.

[10] E. J. Moniz, "Simulating energy technology innovation," American Academy of Arts and Sciences, 2012.

[11] The GridWise Architecture Council (GWAC), "GridWise transactive energy framework," draft version, October 2013.

[12] Pacific Northwest Smart Grid Demonstration Project (PNW-SGDP), http://www.pnwsmartgrid.org/ (accessed, August 1, 2014).

[13] Organization for Advancement of Structured Information Standards (OASIS) standard, "Energy Interoperation Version 1.0,” June 2014.

[14] National Institute of Standads and Technology (NIST) Report, "Guidelines for Smart Grid Cyber Security," NISTR 7228, 2010.

[15] Smart Grid Interoperability Panel (SGIP), Interoperability Process Reference Manual (IPRM), v 2.0, 2012.

[16]

[17] California Energy Commission (CEC), "Building energy efficiency standards for residential and nonresidential buildings," Title 24, 2013.

[18] International Electrotechnical Commission (IEC), "Systems interface between customer energy management system and the power management system - Part 10-1: Open Automated Demand Response (OpenADR 2.0b Profile Specification)," IEC/PAS 62746-10-1 ed1.0, 2014.

[19] M. A Piette, G. Ghatikar, S. Kiliccote, D. Watson, E. Koch, and D. Hennage, "Design and operation of an open, interoperable, automated demand response infrastructure for commercial buildings," LBNL-2340E, JCISE, Vol. 9, Issue 2, June 2009.

[20] American Society of Heating, Refrigerating, and Air conditioning Engineers (ASHRAE), "Facility Smart Grid information model (FSGIM)," Advisory public review draft, July 2012.

[21] The Gridwise ${ }^{\circledR}$ Architecture Council (GWAC); "GridWise interoperability contextsetting framework," 2008.

[22] QualityLogic, Inc., "Transactive control and OpenADR roles and relationships," Revision 1.07, May 2014. 